[21] B. Triggs, P. McLauchlan, R. Hartley, and A. Fitzgibbon, "Bundle adjustment-A modern synthesis," in Vision Algorithms: Theory and Practice, W. Triggs, A. Zisserman, and R. Szeliski, Eds. New York: Springer-Verlag, Lecture Notes in Computer Science, 2000, pp. 298-375.

[22] J. Weng, T. S. Huang, and N. Ahuja, "Motion and structure from two perspective views: Algorithms, error analysis, and error estimation," IEEE Trans. Pattern Anal. Mach. Intell., vol. 11, no. 5, pp. 451-476, May 1989.

[23] Z. Zhang, "Determining the epipolar geometry and its uncertainty: A review," Int. J. Comput. Vis., vol. 27, no. 2, pp. 161-195, 1998.

[24] Z. Zhang, "On the optimization criteria used in two-view motion analysis," IEEE Trans. Pattern Anal. Mach. Intell., vol. 20, no. 7, pp. 717-729, Jul. 1998.

[25] Z. Zhang, R. Deriche, O. Faugeras, and Q. T. Luong, "A robust technique for matching two uncalibrated image through the recovery of the unknown epipolar geometry," Artif. Intell., vol. 78, pp. 87-119, 1995.

\section{Vision-Based Localization for Leader-Follower Formation Control}

Gian Luca Mariottini, Member, IEEE, Fabio Morbidi, Student Member, IEEE, Domenico Prattichizzo, Member, IEEE, Nicholas Vander Valk, Student Member, IEEE, Nathan Michael, Student Member, IEEE,

George Pappas, Fellow, IEEE, and Kostas Daniilidis, Senior Member, IEEE

Abstract-This paper deals with vision-based localization for leaderfollower formation control. Each unicycle robot is equipped with a panoramic camera that only provides the view angle to the other robots. The localization problem is studied using a new observability condition valid for general nonlinear systems and based on the extended output Jacobian. This allows us to identify those robot motions that preserve the system observability and those that render it nonobservable. The state of the leader-follower system is estimated via the extended Kalman filter, and an input-state feedback control law is designed to stabilize the formation. Simulations and real-data experiments confirm the theoretical results and show the effectiveness of the proposed formation control.

Index Terms-Feedback linearization, formation control, mobile robots, nonlinear observability, panoramic cameras.

\section{INTRODUCTION}

\section{A. Motivation and Related Work}

A growing interest in coordination and control of multiple autonomous agents has matured over the past few years [7], [12], [22], [27], [28]. The formation control problem has been playing an important role in this research area, giving rise to a rich literature [2], [15], [17], [30], [31]. By formation control, we simply mean the problem of controlling the relative position and orientation of group of robots while allowing the group to move as a whole. In the leader-follower formation control approach, a robot, i.e., the leader, moves along a predefined trajectory, while the other robots, i.e., the followers, are supposed to
Manuscript received August 28, 2008; revised January 19, 2009, May 28 2009, and August 22, 2009. First published October 30, 2009; current version published December 8, 2009. This paper was recommended for publication by Associate Editor D. Song and Editor L. Parker upon evaluation of the reviewers' comments. This work was supported by the National Science FoundationEnvironmental Industry Associations under Grant 0324977, the National Science Foundation-Information and Intelligent System under Grant 0713260, the National Science Foundation-Industrial Innovation and Partnerships under Grant 0742304, the Department of Defense Multidisciplinary University Research Initiative under Grant 19-02-1-0383, and by the Fondazione Monte dei Paschi di Siena 2005.

G. L. Mariottini is with the Department of Computer Science and Engineering, University of Minnesota, Minneapolis, MN 55455 USA (e-mail: gianluca@cs.umn.edu).

F. Morbidi and D. Prattichizzo are with the Dipartimento di Ingegneria dell'Informazione, University of Siena, Siena 53100, Italy.

N. V. Valk, N. Michael, G. Pappas, and K. Daniilidis are with the General Robotics, Automation, Sensing and Perception Laboratory, University of Pennsylvania, Philadelphia, PA 19104 USA.

This paper has supplementary downloadable multimedia material available at http://ieeexplore.ieee.org provided by the author.

Color versions of one or more of the figures in this paper are available online at http://ieeexplore.ieee.org.

Digital Object Identifier 10.1109/TRO.2009.2032975 
maintain a desired distance from it and orientation to it [10], [13]. Even if leader-follower architectures are known to have poor disturbance rejection properties and the over-reliance on a single agent for achieving a common goal may be undesirable, this approach is appreciated for its simplicity and scalability.

From a sensor viewpoint, cameras have recently received an increasing attention in robotics due to their low cost and the rich information they provide when compared with other traditional sensors (e.g., laser range finders, and sonars). Solving the formation control problem exclusively with on-board vision sensors is challenging, because cameras provide the view-angles to the other robots but not the distance. In this respect, the formation can be controlled only if a localization problem has been solved, i.e., only the data provided by the on-board cameras are used to estimate the relative distance of the robots w.r.t. a common reference frame (e.g., on the leader).

For the localization problem to be solvable, the system must be $o b$ servable. According to control theory, a system is observable when it is possible to reconstruct the initial state by knowing, in a given time interval, the control inputs and the sensor measurements. In particular, when dealing with vision sensors, the localization is intrinsically nonlinear [5], meaning that linearized approximations can be nonobservable, while tools from nonlinear systems theory prove the possibility to reconstruct the state. Such a problem is usually referred to as the observability of perspective dynamical systems. It recently stimulated a great deal of research both in the robotics and control community (see e.g., [1], [9], [14], [19], and the references therein).

In [11], the state-estimation problem for a single robot with onboard camera is approached using a Luenberger-like nonlinear observer based on the projection of stationary landmarks in the environment. In [32], the localization problem for a team of nonholonomic mobile robots with calibrated vision sensors has been solved using motionsegmentation techniques based on optical-flow. In [13], an interesting centralized framework for vision-based leader-follower formation control has been introduced. However, since both the camera-intrinsic calibration parameters and the height of the camera to the floor are supposed a priori known, then the distance between the robots does not need to be estimated and can be computed directly from the camera image. These strong assumptions restrict the practical applicability of the control strategy in [13] to near robots.

\section{B. Contribution}

This paper focuses on leader-follower formations made of unicycle robots, each equipped with an omnidirectional camera. As an improvement over the existing literature, we do not assume to know any camera/mirror calibration parameters (mirror size or focal length), nor the pose of any stationary landmark. Only the view-angle to the other robots is provided by each camera and not the distance that must be estimated. As an original contribution, this paper studies the localization problem using a new observability condition valid for general nonlinear systems and based on the extended output Jacobian (EOJ). In particular, we present a sufficient condition for the observability of the vision-based leader-follower system based on the EOJ. A discussion of the necessary condition of observability is also given. This allows us to identify those robot motions that preserve the observability and those that render it nonobservable.

The state of the system is estimated via the extended Kalman filter (EKF), and an input-state feedback control law is designed to stabilize the formation.

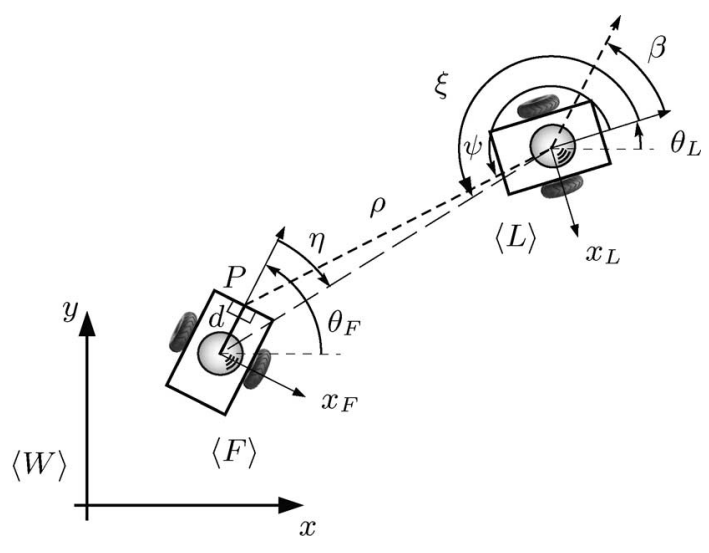

Fig. 1. Leader-follower setup in polar coordinates representation.

Simulations as well as real-data experiments performed with Scarab robots illustrate the robot motions that are critical for system observability and show the effectiveness of the proposed formation control.

This paper is the outgrowth of [24], compared with which we provide herein a more complete study of the nonlinear observability as well as more accurate simulations and experimental results.

\section{Organization}

The rest of the paper is organized as follows. In Section II, the leader-follower kinematic model and the assumptions on sensing and communication are presented. In Section III, we introduce the new observability condition based on the EOJ. In Section IV, the input-state feedback control law is designed. Simulations, as well as experimental results, are presented and discussed in Section V. In Section VI, the main contributions of the paper are summarized and future research directions are highlighted. The Appendix reviews some basic facts on the consistency of a state estimator.

\section{LEADER-FOLLOWER SETUP}

\section{A. Kinematic Model}

Let us consider the leader-follower setup in Fig. 1. The kinematics of each robot can be abstracted as a unicycle model

$$
\dot{x}=v \cos \theta, \quad \dot{y}=v \sin \theta, \quad \dot{\theta}=\omega
$$

where $(x, y)$ represents the position of each robot and $\theta$ its orientation with respect to the world frame $\langle W\rangle$. The leader $\langle L\rangle$ has a configuration vector $\left[\begin{array}{lll}x_{L} & y_{L} & \theta_{L}\end{array}\right]^{T}$, while the follower $\langle F\rangle$ has a vector $\left[\begin{array}{lll}x_{F} & y_{F} & \theta_{F}\end{array}\right]^{T}$. The control inputs of the leader and the follower are the linear and angular velocities $\left[v_{L} \omega_{L}\right]^{T}$ and $\left[\begin{array}{ll}v_{F} & \omega_{F}\end{array}\right]^{T}$, respectively.

The whole leader-follower system can be modeled using polar coordinates, where $\rho$ is the distance from the center of the leader to the marker $P$ placed at a known distance $d$ on the follower (see Fig. 1). The variable $\psi$ is the view-angle from the $y$-axis of the leader to the marker $P$, while $\beta$ is the relative orientation of the robots, i.e., $\beta \triangleq \theta_{L}-\theta_{F}$.

In the spirit of [13] and [24], we introduce here the following kinematic model:

Proposition 1 (Leader-Follower Kinematic Model): With reference to Fig. 1, the leader-follower kinematic model can be written as follows:

$$
\dot{\mathbf{s}}=\mathbf{G}(\mathbf{s}) \mathbf{u}
$$




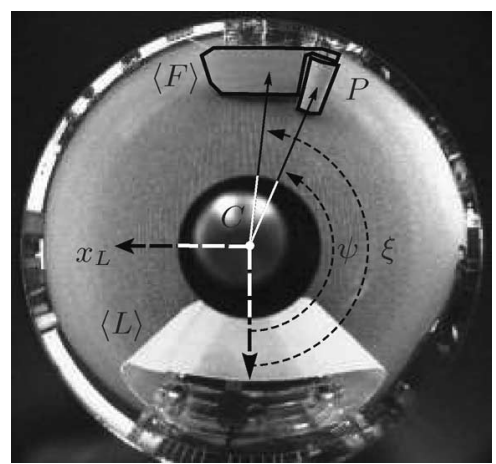

Fig. 2. View-angles computation (on the leader). Hue, Saturation, and Value (HSV) color blob detection is used to determine the two angles $\xi$ and $\psi$ from the leader to the follower's center $C$ and marker $P$, respectively.

where $\mathbf{s} \triangleq\left[\begin{array}{lll}\rho & \psi & \beta\end{array}\right]^{T}, \mathbf{u} \triangleq\left[\begin{array}{llll}v_{F} & \omega_{F} & v_{L} & \omega_{L}\end{array}\right]^{T}$, and

$$
\mathbf{G}(\mathbf{s})=\left[\begin{array}{cccc}
\cos \gamma & d \sin \gamma & -\cos \psi & 0 \\
-\frac{\sin \gamma}{\rho} & \frac{d \cos \gamma}{\rho} & \frac{\sin \psi}{\rho} & -1 \\
0 & -1 & 0 & 1
\end{array}\right]
$$

where $\gamma \triangleq \beta+\psi$.

The kinematic model in the case of $q$ followers can be obtained by simply extending (2). In this case, the input vector is $\mathbf{u} \triangleq\left[\begin{array}{lllllll}v_{F_{1}} & \omega_{F_{1}} & \ldots & v_{F_{q}} & \omega_{F_{q}} & v_{L} & \omega_{L}\end{array}\right]^{T}$ and the state vector is $\mathbf{s} \triangleq\left[\begin{array}{lll}\mathbf{s}_{1}^{T} & \ldots & \mathbf{s}_{q}^{T}\end{array}\right]^{T} \in \mathbb{R}^{3 q}$ (see [24] for more details).

\section{B. Sensing}

Each robot is equipped with only an omnidirectional camera [4]. This sensor is particularly suited for mobile robot navigation because of its field of view, which is wider than that of a standard pinhole camera (see Fig. 2). According to the setup in Fig. 1, $\langle L\rangle$ can measure the viewangles $\xi$ and $\psi$ given by the observation of the follower's centroid and the colored marker $P$, respectively. Analogously, the camera on $\langle F\rangle$ can measure the view-angle $\eta$ to the leader.

The measurement of view-angles is obtained on each robot by means of an automatic real-time color detection and tracking algorithm [16]. Since in our setup each robot and the marker $P$ are characterized by a specific color (see Fig. 5), the leader-follower sensing association is fully automatic. More implementation details are discussed in Section V-B.

\section{Communication}

The state estimation process and the control computation are centralized on the leader, which transmits to the follower the control velocities $\left[\begin{array}{ll}v_{F} & \omega_{F}\end{array}\right]^{T}$ needed to drive the formation. Due to the above assumptions, the inter-robot communication is made fast since the follower only needs to transmit its view-angle $\eta$ to the leader. We assume no communication delays in the view-angle transmission.

From Fig. 1, it is evident that $\beta$ can be computed as follows:

$$
\beta=-\xi+\eta+\pi
$$

To simplify the discussion, we will henceforth refer only to $\beta$, implicitly assuming the transmission of $\eta$. To summarize, the leader can measure a 2-D output vector

$$
\mathbf{y} \triangleq\left[\begin{array}{ll}
y_{1} & y_{2}
\end{array}\right]^{T}=\left[\begin{array}{ll}
\psi & \beta
\end{array}\right]^{T} .
$$

As a concluding remark, it is worth emphasizing here the two peculiar features that differentiate this paper from [13]. First of all, we do not assume a full knowledge of camera calibration parameters. In fact, only the image center $C$ is needed to compute the view-angles and, in many practical cases, $C$ simply coincides with the central black hole in the panoramic image (see Fig. 2). Second, and the most important feature, we assume that the leader-follower relative distance $\rho$ in (2) is unknown. The problem of range estimation from angular measurements is discussed in the next section.

\section{VISION-BASED OBSERVABILITY OF LEADER-FOLLOWER FORMATIONS}

This section reviews some basic facts on the observability of nonlinear systems [20], [21] and presents, in Proposition 3, a new general condition that will be used to study the observability of leader-follower formations.

In Section III-C, we propose an interesting geometrical interpretation of our observability condition. Finally, in Section III-D, we discuss to what extent this condition is also necessary.

\section{A. Observability of Nonlinear Systems}

Consider a generic nonlinear system $\Sigma$ of the form

$$
\Sigma:\left\{\begin{array}{l}
\dot{\mathbf{s}}(t)=\mathbf{f}(\mathbf{s}(t), \mathbf{u}(t)), \quad \mathbf{s}(0) \equiv \mathbf{s}_{0} \\
\mathbf{y}(t)=\mathbf{h}(\mathbf{s}(t))=\left[\begin{array}{llll}
h_{1} & h_{2} & \cdots & h_{m}
\end{array}\right]^{T}
\end{array}\right.
$$

where $\mathbf{s}(t)=\left[s_{1}(t) s_{2}(t) \ldots s_{n}(t)\right]^{T} \in \mathcal{S}$ is the state, $\mathbf{y}(t) \in \mathcal{Y}$ the measurements vector, and $\mathbf{u}(t) \in \mathcal{U}$ the input vector. $\mathcal{S}, \mathcal{Y}$, and $\mathcal{U}$ are differential manifolds of dimensions $n, m$, and $p$, respectively.

Let $\mathbf{y}\left(t, \mathbf{s}_{0}, \mathbf{u}\right) \triangleq \mathbf{h}\left(\mathbf{s}\left(t, \mathbf{s}_{0}, \mathbf{u}\right)\right)$ denote the output of $\Sigma$ at time $t$, for input $\mathbf{u}$ and initial state $\mathbf{s}_{0}$. Two states $\mathbf{s}_{1}$ and $\mathbf{s}_{2} \in \mathcal{S}$ are said to be indistinguishable (denoted $\mathbf{s}_{1} I \mathbf{s}_{2}$ ) for $\Sigma$ if for every admissible input function $\mathbf{u}$, the output function $\mathbf{y}\left(t, \mathbf{s}_{1}, \mathbf{u}\right), t \geq 0$ of the system for initial state $\mathbf{s}_{1}$ and the output function $\mathbf{y}\left(t, \mathbf{s}_{2}, \mathbf{u}\right), t \geq 0$ of the system for initial state $\mathbf{s}_{2}$ are identical on their common domain of definition [26] (if, in addition, the trajectories of the system originating from $\mathbf{s}_{1}$ and $\mathbf{s}_{2}$ lie in a subset $\mathcal{M}$ of $\mathcal{S}$, then $\mathbf{s}_{1}$ and $\mathbf{s}_{2}$ are said to be $\mathcal{M}$-indistinguishable and denoted by $\left.\mathbf{s}_{1} I_{\mathcal{M}} \mathbf{s}_{2}\right)$. Note that the indistinguishability is an equivalence relation on $\mathcal{S}$. The notions of observability and indistinguishability are tightly connected, as shown in the next definition [18].

Definition 1 (Observability): System $\Sigma$ in (5) is said to be observable at $\mathbf{s}_{0} \in \mathcal{S}$ if $I\left(\mathbf{s}_{0}\right)=\left\{\mathbf{s}_{0}\right\}$.

For a generic nonlinear system, as the one in (5), global or complete observability cannot be usually expected and the more specific notion of local weak observability has been introduced in [18]. This notion also has the advantage of lending itself to a simple algebraic test of observability.

Definition 2 (Local Weak Observability): $\Sigma$ is said to be locally weakly observable at $\mathbf{s}_{0} \in \mathcal{S}$ if there exists an open neighborhood $\mathcal{M}$ of $\mathbf{s}_{0} \in \mathcal{S}$ such that, for every open neighborhood $\mathcal{V}$ of $\mathbf{s}_{0}$ contained in $\mathcal{M}, I_{\mathcal{V}}\left(\mathbf{s}_{0}\right)=\left\{\mathbf{s}_{0}\right\}$.

Intuitively, $\Sigma$ is locally weakly observable at $\mathbf{s}_{0}$ if one can instantaneously distinguish $\mathbf{s}_{0}$ from its neighbors.

Before we proceed any further, let us introduce two differential operators that will be useful in the next derivations.

Given a scalar-valued function $\lambda(\mathbf{s}): \mathbb{R}^{n} \rightarrow \mathbb{R}$, the gradient of $\lambda$ is defined as follows:

$$
d \lambda(\mathbf{s}) \triangleq \frac{\partial \lambda(\mathbf{s})}{\partial \mathbf{s}}=\left[\begin{array}{llll}
\frac{\partial \lambda(\mathbf{s})}{\partial s_{1}} & \frac{\partial \lambda(\mathbf{s})}{\partial s_{2}} & \cdots & \frac{\partial \lambda(\mathbf{s})}{\partial s_{n}}
\end{array}\right] .
$$


The Lie derivative of a scalar-valued function $h(\mathbf{s})$ along a vector field $\mathbf{g}: \mathbb{R}^{n} \rightarrow \mathbb{R}^{n}$ is a real-valued function, which is defined as $L_{\mathbf{g}} h(\mathbf{s}) \triangleq d h \mathbf{g}$. The Lie derivative can be recursively repeated as

$$
L_{\mathbf{g}}^{k} h(\mathbf{s}) \triangleq L_{\mathbf{g}}\left[L_{\mathbf{g}}^{k-1} h(\mathbf{s})\right], \forall k \geq 1
$$

being $L_{\mathbf{g}}^{0} h(\mathbf{s}) \triangleq h(\mathbf{s})$.

A sufficient condition for the local weak observability of $\Sigma$ has been first introduced in [18] and is reported here for the reader's convenience.

Proposition 2 (Observability Rank Condition): System $\Sigma$ in (5) is locally weakly observable at a point $\mathbf{s}_{0} \in \mathcal{S}$, if there exists an open neighborhood $\mathcal{M}$ of $\mathbf{s}_{0}$ and positive integers $j_{1}, j_{2}, \ldots, j_{m}$ satisfying $j_{1}+j_{2}+\cdots+j_{m}=n$ such that, for arbitrary $\mathbf{s} \in \mathcal{M}$, the observability matrix $\mathcal{O}$ defined as the matrix with rows

$$
\mathcal{O} \triangleq\left\{L_{\mathbf{f}}^{j-1} d h_{i}(\mathbf{s}) \mid i=1, \ldots, m ; j=1, \ldots, j_{m}\right\}
$$

is full rank.

An equivalent and more intuitive formulation of Proposition 2, that relies on the EOJ [11], is presented in the next proposition.

Proposition 3 (EOJ Observability Rank Condition [24]): System $\Sigma$ is locally weakly observable at a point $\mathbf{s}_{0} \in \mathcal{S}$, if there exists an open neighborhood $\mathcal{M}$ of $\mathbf{s}_{0}$ such that, for arbitrary $\mathbf{s} \in \mathcal{M}$, the EOJ $\mathbf{J} \in \mathbb{R}^{m n \times n}$ defined as the matrix with rows

$$
\mathbf{J} \triangleq\left\{d h_{i}^{(j-1)}(\mathbf{s}) \mid i=1, \ldots, m ; j=1, \ldots, n\right\}
$$

is full rank. The superscript $j$ refers to the order of time differentiation of the functions $h_{i}(\mathbf{s})$.

Proof: The proof is constructive. Computing the Lie derivatives in (6), it turns out that for $i=1, \ldots, m$

$$
\begin{aligned}
(j=1) L_{\mathbf{f}}^{0} d h_{i}(\mathbf{s}) & =\frac{\partial h_{i}(\mathbf{s})}{\partial \mathbf{s}}=d h_{i}^{(0)}(\mathbf{s}) \\
(j=2) L_{\mathbf{f}}^{1} d h_{i}(\mathbf{s}) & =\frac{\partial}{\partial \mathbf{s}}\left[L_{\mathbf{f}}^{0} d h_{i}(\mathbf{s})\right] \mathbf{f}(\mathbf{s}) \\
& =d\left[\frac{\partial h_{i}}{\partial \mathbf{s}} \mathbf{f}(\mathbf{s})\right]=d\left[\frac{\partial h_{i}}{\partial \mathbf{s}} \frac{\partial \mathbf{s}}{\partial t}\right]=d h_{i}^{(1)}(\mathbf{s}) \\
(j=3) L_{\mathbf{f}}^{2} d h_{i}(\mathbf{s})= & d\left[\frac{\partial}{\partial \mathbf{s}}\left[L_{\mathbf{f}}^{1} h_{i}\right] \mathbf{f}(\mathbf{s})\right] \\
= & d\left[\frac{\partial h_{i}^{(1)}}{\partial \mathbf{s}} \dot{\mathbf{s}}\right]=d\left[\frac{\partial\left(\frac{\partial h_{i}}{\partial t}\right)}{\partial \mathbf{s}} \frac{\partial \mathbf{s}}{\partial t}\right]=d h_{i}^{(2)}(\mathbf{s}) \\
\vdots & \vdots \\
(j=n) L_{\mathbf{f}}^{n-1} d h_{i}(\mathbf{s}) & =d h_{i}^{(n-1)}(\mathbf{s})
\end{aligned}
$$

and by stacking the vectors (8)-(11) in a matrix, from Proposition 2, we obtain the thesis.

Remark 1: Proposition 3 states that the observability of $\Sigma$ can be tested by checking the rank of the EOJ $\mathbf{J}$, which is made of the state partial derivatives of the output vector and of all its $n-1$ time derivatives. In particular, $\Sigma$ is locally weakly observable also when at least one $n \times n$ submatrix of $\mathbf{J}$ is full rank.

\section{B. Observability of Leader-Follower Formations}

Proposition 3 is used here to determine an observability condition for the leader-follower system described in Section II. From this proposition, the observability of (2) with output in (4) is guaranteed when at

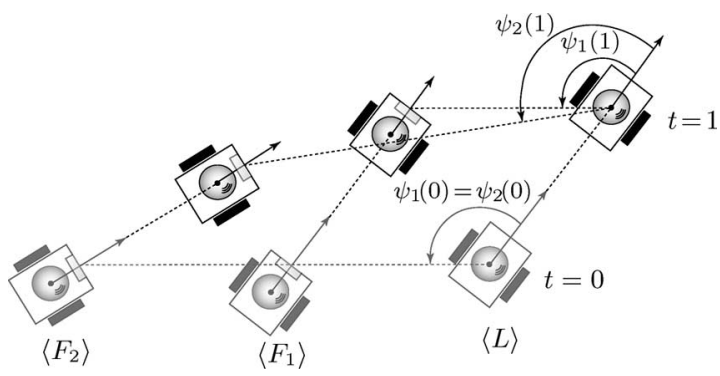

Fig. 3. Geometrical interpretation of the EOJ singularity.

least one $3 \times 3$ submatrix of the whole $6 \times 3 \mathrm{EOJ}$ is nonsingular. Let us consider, e.g., the submatrix $\overline{\mathbf{J}}$

$$
\overline{\mathbf{J}}=\left[\begin{array}{ccc}
\frac{\partial y_{1}}{\partial \rho} & \frac{\partial y_{1}}{\partial \psi} & \frac{\partial y_{1}}{\partial \beta} \\
\frac{\partial \dot{y}_{1}}{\partial \rho} & \frac{\partial \dot{y}_{1}}{\partial \psi} & \frac{\partial \dot{y}_{1}}{\partial \beta} \\
\frac{\partial y_{2}}{\partial \rho} & \frac{\partial y_{2}}{\partial \psi} & \frac{\partial y_{2}}{\partial \beta}
\end{array}\right]=\left[\begin{array}{ccc}
0 & 1 & 0 \\
\frac{\partial \dot{\psi}}{\partial \rho} & \frac{\partial \dot{\psi}}{\partial \psi} & \frac{\partial \dot{\psi}}{\partial \beta} \\
0 & 0 & 1
\end{array}\right]
$$

whose determinant is

$$
\operatorname{det}(\overline{\mathbf{J}})=-\frac{\partial \dot{\psi}}{\partial \rho}=\frac{1}{\rho}\left[\dot{\psi}+\omega_{L}\right] .
$$

Therefore, if $\dot{\psi}+\omega_{L} \neq 0$, the system is observable.

In the case of $q>1$ followers, the observability condition is a simple extension of (13) (see [24]).

\section{Geometrical Interpretation of the EOJ Singularity}

In Fig. 3, we provide a simple example to give some geometrical insight into the condition found in the previous section. A leader and two followers are considered at two different time instants: $t=0$ and $t=1$. All the robots move with the same translational velocity and zero angular velocity.

We first note that $\psi_{2}(1) \neq \psi_{2}(0)$ (and thus $\dot{\psi}_{2} \neq 0$ ) due to the different initial orientation between $\langle L\rangle$ and $\left\langle F_{2}\right\rangle$. Then, from (13), it turns out that the state $\mathbf{s}_{2}$ is observable. This is intuitively correct, since the visual information varies in time and it is then expected to improve the localizability. This also leads to the intuition that curvilinear trajectories have a favorable effect on observability, since a change in the output signal (4) occurs there.

From an inspection of Fig. 3, it is also evident that there is not any improvement in the localization between $\langle L\rangle$ and $\left\langle F_{1}\right\rangle$, since their relative motion is zero from $t=0$ to $t=1$ (and then, $\dot{\psi}_{1}=0$ ). At this point, one could guess that the state $\mathbf{s}_{1}$ is not observable. To confirm this intuition, we should verify to what extent the condition of Proposition 3 is also necessary. This point is discussed in full detail in the next section.

\section{Necessary Condition of Observability: Discussion}

Proposition 2 provides a sufficient condition for the local weak observability of $\Sigma$. However, as pointed out in [18, Th. 3.11], the converse implication is "almost" true. This means that if $\Sigma$ is locally weakly observable, then the observability rank condition is satisfied generically.

"Generically" means that the observability matrix $\mathcal{O}$ in (6), and equivalently $\mathbf{J}$ in (7), must be full rank everywhere, except possibly within a subset of the domain of $\mathbf{s}$ [8]. Algebraically, the check for observability can be done by testing that $\mathbf{J}$ is not full rank for all values of $\mathbf{s}$, which will then imply that $\Sigma$ is not locally weakly observable. 
In the case of a single follower, the general expression for $\mathbf{J} \in \mathbb{R}^{6 \times 3}$ is

$$
\mathbf{J}^{T}=\left[\begin{array}{llllll}
\frac{\partial y_{1}}{\partial \rho} & \frac{\partial \dot{y}_{1}}{\partial \rho} & \frac{\partial \ddot{y}_{1}}{\partial \rho} & \frac{\partial y_{2}}{\partial \rho} & \frac{\partial \dot{y}_{2}}{\partial \rho} & \frac{\partial \ddot{y}_{2}}{\partial \rho} \\
\frac{\partial y_{1}}{\partial \psi} & \frac{\partial \dot{y}_{1}}{\partial \psi} & \frac{\partial \ddot{y}_{1}}{\partial \psi} & \frac{\partial y_{2}}{\partial \psi} & \frac{\partial \dot{y}_{2}}{\partial \psi} & \frac{\partial \ddot{y}_{2}}{\partial \psi} \\
\frac{\partial y_{1}}{\partial \beta} & \frac{\partial \dot{y}_{1}}{\partial \beta} & \frac{\partial \ddot{y}_{1}}{\partial \beta} & \frac{\partial y_{2}}{\partial \beta} & \frac{\partial \dot{y}_{2}}{\partial \beta} & \frac{\partial \ddot{y}_{2}}{\partial \beta}
\end{array}\right]
$$

As also shown in Fig. 3, if, $\langle L\rangle$ and $\left\langle F_{1}\right\rangle$ move along straight trajectories, then $\mathbf{y}$ is constant; hence $\dot{\mathbf{y}}=\ddot{\mathbf{y}}=\mathbf{0}$. From (14), this implies that $\mathbf{J}$ has only two nonzero rows, i.e., its rank can never be 3 . Therefore, the state $s_{1}$ of the system is not locally weakly observable exactly when the robots move along straight trajectories.

For a system not to be observable means that the output does not convey an information rich enough to allow the observer to provide a correct estimate of the state, thus negatively affecting the control action.

Such an undesired effect on the control has been observed in both the simulation and experimental results, as discussed in Section V-A and $\mathrm{B}$.

\section{E. Observer Design}

In order to control the formation, an estimate $\hat{\mathbf{s}}$ of the true state $\mathbf{s}$ is required. An EKF has been designed to estimate the state s, given the input vector $\mathbf{u}$ and the output $\mathbf{y}$. We assume additive noise in both the process and measurement equations

$$
\begin{aligned}
& \dot{\mathbf{s}}=\mathbf{G}(\mathbf{s}) \mathbf{u}+\mathbf{z} \\
& \mathbf{y}=\mathbf{C s}+\mathbf{v}
\end{aligned}
$$

where $\mathbf{C}$ is the output transition matrix, and $\mathbf{z}$ and $\mathbf{v}$ are zero mean white Gaussian noises with covariance matrices $\mathbf{Q}$ and $\mathbf{R}$, respectively. $\mathbf{s}(0), \mathbf{z}$, and $\mathbf{v}$ are assumed to be uncorrelated. Equation (15) has been discretized using the Euler forward method with sampling time $T_{c}$

$$
\mathbf{s}(k+1)=\boldsymbol{\Gamma}(\mathbf{s}(k), \mathbf{u}(k))+T_{c} \mathbf{z}
$$

where $\boldsymbol{\Gamma}(\mathbf{s}(k), \mathbf{u}(k)) \triangleq T_{c} \mathbf{G}(\mathbf{s}) \mathbf{u}+\mathbf{s}(k)$, and $k \in \mathbb{N}$.

\section{INPUT-STATE FEEDBACK CONTROL}

Consider the set of kinematic equations equivalent to (2)

$$
\begin{aligned}
\dot{\mathbf{s}}_{r} & =\mathbf{F}(\mathbf{s}) \mathbf{u}_{L}+\mathbf{H}(\mathbf{s}) \mathbf{u}_{F} \\
\dot{\beta} & =\omega_{L}-\omega_{F}
\end{aligned}
$$

where $\mathbf{s}_{r} \triangleq\left[\begin{array}{ll}\rho & \psi\end{array}\right]^{T}$ is the reduced state-space vector. Matrices $\mathbf{H}$ and $\mathbf{F}$ are the two upper-left and right $2 \times 2$ submatrices of $\mathbf{G}$, respectively.

In the spirit of [13], we propose here an input-state feedback control law for the robot formation. Let us consider the following control input:

$$
\mathbf{u}_{F} \triangleq\left[\begin{array}{ll}
v_{F} & \omega_{F}
\end{array}\right]^{T}=\mathbf{H}^{-1}(\mathbf{s})\left(\mathbf{p}-\mathbf{F}(\mathbf{s}) \mathbf{u}_{L}\right)
$$

where

$$
\mathbf{p}=\dot{\mathbf{s}}_{r}^{\text {des }}-\mathbf{K}\left(\mathbf{s}_{r}-\mathbf{s}_{r}^{\text {des }}\right)
$$

and $\mathbf{K}=\operatorname{diag}\left\{k_{1}, k_{2}\right\}$, with $k_{1}, k_{2}>0$. The superscript "des" refers to the desired state. Equation (19) acts in (17) as a feedback linearizing control, so that the closed-loop dynamics becomes

$$
\dot{\mathbf{s}}_{r}=\dot{\mathbf{s}}_{r}^{\mathrm{des}}-\mathbf{K}\left(\mathbf{s}_{r}-\mathbf{s}_{r}^{\mathrm{des}}\right), \quad \dot{\beta}=\omega_{L}-\omega_{F} .
$$

The following proposition states that it is sufficient to control $\mathbf{s}_{r}$ toward $\mathbf{s}_{r}^{\text {des }}$ using (19) to guarantee the local stability of the whole statespace vector s. Due to the physical constraints of the robots, we will reasonably assume that the angular velocity of the leader is bounded.

Proposition 4: Let us suppose that $v_{L}>0,\left|\omega_{L}\right|<W_{\max },|\beta(0)|<\pi$, and $\dot{\mathbf{s}}_{r}^{\text {des }}$ is bounded. Then the control law (19)-(20) stabilizes the system dynamics (17)-(18).

Proof: Let us refer to $\left[\begin{array}{ll}e_{\rho} & e_{\psi}\end{array}\right]^{T}=\mathbf{s}_{r}-\mathbf{s}_{r}^{\text {des }}$ as the state tracking error vector. From (23), it follows that $\left[\begin{array}{ll}e_{\rho} & e_{\psi}\end{array}\right]^{T}$ is globally exponentially stable. We now prove that the internal dynamics is stable, i.e., that $|\beta|$ is bounded. Drawing $\omega_{F}$ from (19), (18) can be rewritten as

$$
\begin{aligned}
\dot{\beta}=-\frac{\sin \gamma}{d}\left(\dot{\rho}^{\mathrm{des}}-k_{1} e_{\rho}\right) & -\frac{\rho \cos \gamma}{d}\left(\dot{\psi}^{\mathrm{des}}-k_{2} e_{\psi}\right) \\
& -\frac{v_{L}}{d} \sin \beta-\omega_{L}\left(\frac{\rho}{d} \cos \gamma-1\right) .
\end{aligned}
$$

Since $\omega_{L}$ and $\dot{\mathbf{s}}_{r}^{\text {des }}$ are bounded by hypothesis, (22) can be rewritten as

$$
\dot{\beta}=-\frac{v_{L}}{d} \sin \beta-B(t) .
$$

Note that without the term $B(t)$, a bounded persistent disturbance, (23) is locally asymptotically stable for $|\beta|<\pi$. From the stability theory of nonlinear systems with persistent disturbances [29], where $|\beta(0)|<\pi$ and $B(t)$ are bounded, it follows that $|\beta(t)|<\varepsilon, \forall t>T$, for finite time $T$ and $\forall \varepsilon>0$.

Remark 2 (Distant Robots): Note that if the distance between the leader and the follower is big, it is in general difficult to exactly locate the marker $P$ in the image (see Section II-B and Fig. 2). A possible solution to this problem consists in detecting only the robot centroid, which is equivalent to assuming that $d=0$.

Note that this assumption does not affect the observability condition presented in Section III-B and does not prevent us from using the EKF.

However, it has a negative effect on the control. In fact, $\mathbf{H}(\mathbf{s})$ becomes singular when $d=0$ and the control in (19) is no longer applicable. In [23], we have proposed a feedback control via dynamic extension that allows us to overcome this control issue.

\section{Simulation AND EXPERIMENTAL RESUlts}

\section{A. Simulations}

Simulations have been performed in order to test the validity of the observability analysis in Section III. The following velocity input has been assigned to the leader

$$
\begin{aligned}
& v_{L}(t)=0.3 \mathrm{~m} / \mathrm{s} \\
& \omega_{L}(t)= \begin{cases}0 \mathrm{rad} / \mathrm{s}, & \text { if } t \in\{[0,6],(14,20],(28,34]\} \\
\pi / 8 \mathrm{rad} / \mathrm{s}, & \text { otherwise }\end{cases}
\end{aligned}
$$

which undergoes a piecewise rectilinear-circular trajectory that is particularly suited for checking the observability conditions discussed in Section III-B and D.

The formation considered consists of two followers. We

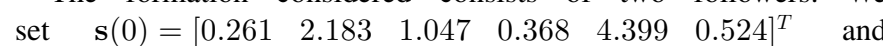
$\mathbf{s}_{r}^{\text {des }}=\left[\begin{array}{llll}0.3 & 3 \pi / 4 & 0.3 & 5 \pi / 4\end{array}\right]^{T}$, where distances are in meters and angles in radians.

The gain matrix of the controller is $\mathbf{K}=6 \mathbf{I}_{4}$, where $\mathbf{I}_{4}$ denotes the $4 \times 4$ identity matrix. The EKF was initialized with $\hat{\mathbf{s}}(0 \mid-1)=\left[\frac{3}{2} \rho_{1}(0) \psi_{1}(0) \beta_{1}(0) \frac{3}{2} \rho_{2}(0) \psi_{2}(0) \beta_{2}(0)\right]^{T}$ corresponding to a $50 \%$ perturbation of the unknown distances to the leader and covariance matrix $\mathbf{P}(0 \mid-1)=10^{-2} \times$ $\operatorname{diag}\{1,1.1,1.1,1,1.1,1.1\}$. The other parameters are $T_{c}=$ 


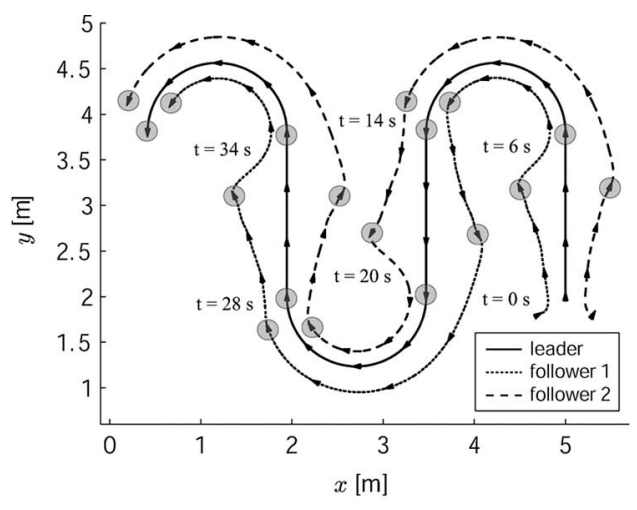

(a)

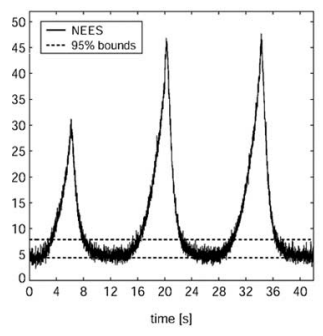

(b)

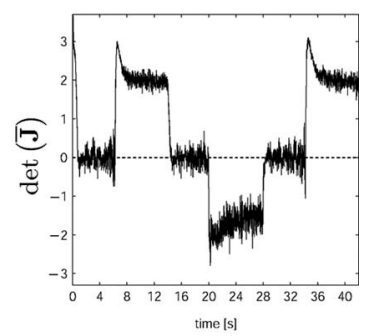

(c)
Fig. 4. Simulation results. (a) Trajectory of the robots. (b) Time history of NEES and $95 \%$ bounds (15 trials). (c) Time history of $\operatorname{det}(\overline{\mathbf{J}})$.

$10 \mathrm{~ms}, d=0.1 \mathrm{~m}, \mathbf{Q}=\operatorname{diag}\left\{3 \times 10^{-5}, \varrho, \varrho, 3 \times 10^{-5}, \varrho, \varrho\right\}$, and $\mathbf{R}=\varrho \mathbf{I}_{4}$, where $\varrho=0.9187 \times 10^{-4} \mathrm{rad}^{2}$. White Gaussian noise has been injected into the measurements.

Fig. 4(a) shows the resulting trajectories of the three robots (the vehicles are drawn every $2 \mathrm{~s}$ in order to have a temporal reference). According to the results described in Section III-D, it is evident that the followers miss the formation exactly along the rectilinear tracts of the trajectory (e.g., in $t \in(14,20])$, where, since visual data are not changing sensibly, the system is not observable. On the other hand, when the leader switches from the rectilinear to the curvilinear tracts (e.g., in $t \in(20,28])$, a change in the visual information occurs, thus leading to an improvement of the localization: The desired formation is, in fact, recovered soon after.

In order to validate the above observations on more trials, we also studied the consistency of the EKF examining the time history of normalized estimation error squared (NEES), which is a concise representation of the estimation error (see the Appendix). Comparing Fig. 4(a) with (b) (here, $r_{1}=4.38, r_{2}=7.87$, and $N=15$ ), it is evident that the NEES tends to leave the $95 \%$ bounds exactly in correspondence of the rectilinear tracts of the leader trajectory (e.g., in $t \in(14,20])$.

Fig. 4(c) reports the time history of $\operatorname{det}(\overline{\mathbf{J}})$, where $\overline{\mathbf{J}}$ is here relative to follower 1 (similar results are obtained with $\overline{\mathbf{J}}$ relative to follower 2 ). According to condition (13), we see that the state $\mathbf{s}_{1}$ (analogously $\mathbf{s}_{2}$ ) is observable along the curvilinear tracts of the trajectory, i.e., where $\operatorname{det}(\overline{\mathbf{J}}) \neq 0$. Moreover, $\operatorname{det}(\overline{\mathbf{J}})$ is near zero exactly along the straight tracts of the trajectory that also correspond to the time intervals in which the NEES increases. This confirms the observability results of Section III-D, for which the state $\mathbf{s}_{1}$ (analogously $\mathbf{s}_{2}$ ) is not observable.

\section{B. Experiments}

In order to validate the proposed formation control strategy in a real scenario, some experiments have been carried out at the General Robotics, Automation, Sensing, and Perception (GRASP) Laboratory,

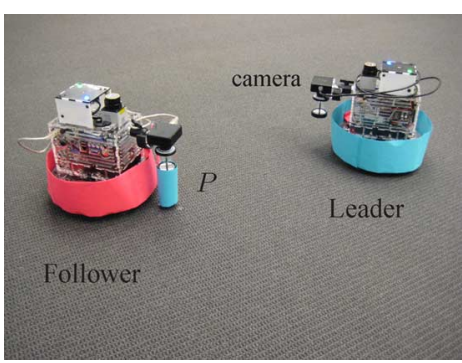

Fig. 5. Experimental setup. The Scarab robots used in the experiments.

University of Pennsylvania, Philadelphia, PA. The experimental setup consists of two Scarab robots acting as a leader and a follower (see Fig. 5). Only one follower has been used in our experiments in order to make the observability analysis as simple as possible. Actually, due to the special communication protocol adopted (cf., Section II-C), we experienced no significant performance change in the localization and formation control when multiple followers were used.

The Scarab is a differential-driven robotic platform designed at the GRASP Laboratory, measuring $20 \times 13.5 \times 22.2 \mathrm{~cm}^{3}$. The leader and the follower run identical modularized software with well-defined interfaces connecting modules via the Player robot architecture system. In order to provide a ground truth information of the actual robot pose, a tracking system consisting of LED markers on the top of each robot and eight overhead cameras are employed. More technical details on Scarab robots and on the tracking system can be found in [25].

The robots are uniquely identified by colored markers and are equipped with a panoramic camera, consisting of a hyperbolic remote reality mirror (folded) screwed onto a Point Grey Firefly IEEE 1394 camera. The image resolution is $320 \times 240$ pixels. Only the image principal point $\left(u_{0}, v_{0}\right)$ is known and is given by $(159.48,123.70)$ pixels and $(172.89,126.53)$ pixels for the leader and the follower's camera, respectively. HSV color blob detection is run on each robot using Intel's OpenCV libraries. Even though more sophisticated visual contour tracking algorithms are available in the literature [6], with our blob detector, we experienced a good compromise between real-time performances, robustness to changing in illumination, partial occlusions, and tracking of far robots. The distance between the center of the robot and the marker is $d=20 \mathrm{~cm}$.

Note that, due to the presence of the LED markers on the top of each robot, the position of the panoramic camera on the vehicles is different from that shown in Fig. 1. However, a simple algebraic transformation is sufficient to readapt the robots' angle measurement to the model in Fig. 1. This transformation has been implemented in the code running on the robots without significantly affecting the performance and the computational load.

For the experiment, we chose $\mathbf{s}(0)=\hat{\mathbf{s}}(0 \mid-1)=\left[\begin{array}{lll}0.75 & 5 / 4 \pi & 0\end{array}\right]^{T}$ and $\mathbf{s}_{r}^{\text {des }}=\left[\begin{array}{ll}0.5 & 5 / 4 \pi\end{array}\right]^{T}$. The control gains are $k_{1}=k_{2}=0.5$ and $T_{c}=0.1$ s. Moreover, $\mathbf{P}(0 \mid-1)=10^{-2} \times \mathbf{I}_{3}, \mathbf{Q}=10^{-5} \times$ $\operatorname{diag}\{3,9,9\}$, and $\mathbf{R}=10^{-5} \times \operatorname{diag}\{9.1,9.1\}$.

As in Section V-A, we selected an input vector $\left[\begin{array}{ll}v_{L} & w_{L}\end{array}\right]^{T}$ that gives rise to a rectilinear/circular trajectory. Other experiments have been performed with different leader trajectories and desired formations, all demonstrating a good robustness of the proposed formation control strategy. However, due to a lack of space, they are not reported here. ${ }^{1}$

Fig. 6(a) shows the trajectory of the robots in the first experiment, from which we can see that the robots are able to achieve the desired formation. The time instants in which the leader switches form the

\footnotetext{
${ }^{1}$ A video with three trials is available as multimedia attachment on IEEE Xplore.
} 


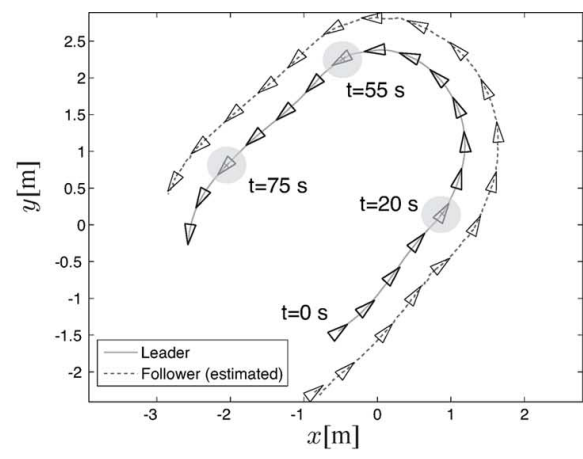

(a)

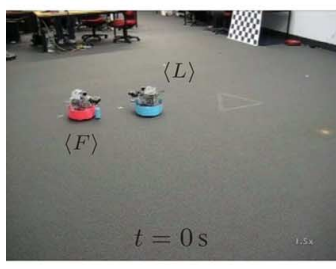

(b)

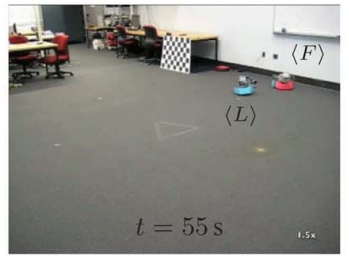

(d)

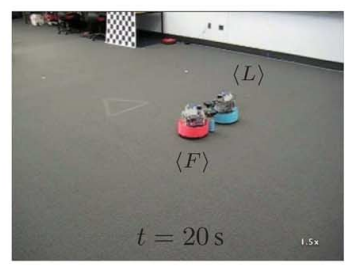

(c)

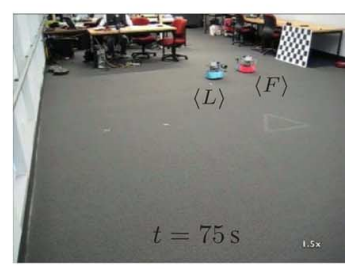

(e)

Fig. 6. Experimental results. (a) Trajectory of the leader and the follower. The time instants in which the leader switches from the rectilinear to the circular tracts, and vice versa, are highlighted. (b)-(e) Snapshots from the experiment.

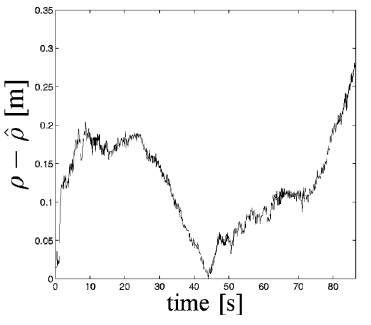

(a)

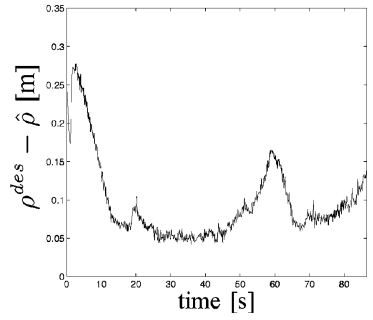

(b)

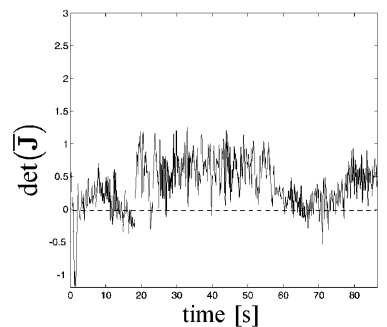

(c)

Fig. 7. Experimental results. Time history of (a) observation error $\rho-\hat{\rho}$, (b) control error $\rho^{\text {des }}-\hat{\rho}$, and (c) $\operatorname{det}(\overline{\mathbf{J}})$.

linear to the curvilinear trajectory, and vice versa, have been highlighted in the figure. A series of snapshots from the experiment is reported in Fig. 6(b)-(e). The range estimation error $\rho-\hat{\rho}$ and the range-tracking error $\rho^{\text {des }}-\hat{\rho}$ are shown in Fig. 7(a) and (b), respectively. The errors on $\psi$ and $\beta$ are not reported here because, differently from the distance $\rho$, these components of the state vector are directly available in $\mathbf{y}$ and are not critical to estimate. As expected, the estimation and tracking errors in Fig. 6(b)-(e) decrease and remain close to zero approximately in $t \in[20,55]$, corresponding to the circular tract of the trajectory. The value of $\operatorname{det}(\overline{\mathbf{J}})$ in Fig. 7(c) is close to zero approximately at the same time instants in which both the tracking and estimation errors increase (e.g., for $t \in(55,70])$, that is, when the leader moves along the rectilinear tracts. This again confirms the results on observability presented in Section III-B and D.

\section{CONCLUSION AND FUTURE WORK}

This paper studies the vision-based localization and control of a leader-follower formation of nonholonomic mobile robots. Each robot is equipped with a panoramic camera that only provides the view-angle to the other vehicles. As an original contribution, we present a new observability condition based on the EOJ that is used to provide a sufficient condition for the observability of the vision-based leader-follower system. A discussion of the necessary condition of observability is also given. This allows us to identify those robot motions that preserve the observability and those that render the system nonobservable.

The state of the leader-follower system is estimated via the EKF, and an input-state feedback control law is designed to stabilize the formation. Simulations and real-data experiments performed with Scarab robots illustrate the theory and show the effectiveness of the proposed formation control.

Future research directions include the study of a decentralized formation-control strategy and the integration of different sensor typologies, such as laser range finders and inertial measurement units.

\section{APPENDIX}

\section{CONSISTENCY OF A STATE ESTIMATOR}

Definition 3: A state estimator is said to be consistent [3] if its stateestimation error $\tilde{\mathbf{s}}(k \mid k) \triangleq \hat{\mathbf{s}}(k)-\mathbf{s}(k \mid k)$ is such that $\mathrm{E}[\tilde{\mathbf{s}}(k \mid k)]=\mathbf{0}$ and $\mathrm{E}\left[\tilde{\mathbf{s}}(k \mid k) \tilde{\mathbf{s}}(k \mid k)^{T}\right]=\mathbf{P}(k \mid k)$.

To practically evaluate the consistency of an estimator, the NEES is defined as

$$
\varepsilon(k) \triangleq \tilde{\mathbf{s}}(k \mid k)^{T} \mathbf{P}^{-1}(k \mid k) \tilde{\mathbf{s}}(k \mid k) .
$$

Let us consider $N$ Monte Carlo simulations that provide $N$ samples $\varepsilon_{i}(k)$ of the random variable $\varepsilon(k)$. Let $\bar{\varepsilon}(k)=1 / N \sum_{i=1}^{N} \varepsilon_{i}(k)$ be the sample mean of $\varepsilon(k)$. The hypothesis that the state estimation errors are consistent with the estimator calculated covariances is not invalidated if $\bar{\varepsilon}(k) \in\left[r_{1}, r_{2}\right], r_{1}, r_{2} \in \mathbb{R}$. Under the Gaussian assumption $N \bar{\varepsilon}(k) \sim \chi_{N 3 q}^{2}$, where $\chi_{N 3 q}^{2}$ is an $N 3 q$ degrees of freedom chi-square distribution. $r_{1}, r_{2}$ can then be computed from a table providing the points on the chi-square distribution for a given tail probability (see e.g., [3, App. C]).

It is worth recalling that even if it is specifically designed for linear systems, the consistency criterion based on the NEES is commonly used in the nonlinear case as well.

\section{REFERENCES}

[1] R. Abdursul, H. Inaba, and B. K. Ghosh, "Nonlinear observers for perspective time-invariant linear systems," Automatica, vol. 40, no. 3, pp. 481490, 2004.

[2] T. Balch and R. C. Arkin, "Behavior-based formation control for multirobot teams," IEEE Trans. Robot. Autom., vol. 14, no. 6, pp. 926-939, Dec. 1998.

[3] Y. Bar-Shalom, X. R. Li, and T. Kirubarajan, Estimation with Applications to Tracking and Navigation. New York: Wiley, 2001.

[4] R. Benosman and S. B. Kang, Panoramic Vision: Sensors, Theory and Applications. New York: Springer-Verlag, 2001.

[5] A. Bicchi, D. Prattichizzo, A. Marigo, and A. Balestrino, "On the observability of mobile vehicles localization," in Proc. IEEE Mediterrean Conf. Control Syst., 1998, pp. 142-147. 
[6] A. Blake and M. Isard, Active Contours. New York: Springer-Verlag, 1998.

[7] F. Bullo, J. Cortes, S. Martinez, Distributed Control of Robotic Networks (Applied Mathematics Series). Princeton, NJ: Princeton Univ. Press, 2009. Available: http://coordinationbook.info.

[8] J. L. Casti, "Recent developments and future perspectives in nonlinear system theory," SIAM Rev., vol. 24, no. 3, pp. 301-331, 1982.

[9] X. Chen and H. Kano, "A new state observer for perspective systems," IEEE Trans. Autom. Control, vol. 47, no. 4, pp. 658-663, Apr. 2002.

[10] L. Consolini, F. Morbidi, D. Prattichizzo, and M. Tosques, "Leader follower formation control of nonholonomic mobile robots with input constraints," Automatica, vol. 44, no. 5, pp. 1343-1349, 2008.

[11] F. Conticelli, A. Bicchi, and A. Balestrino, "Observability and nonlinear observers for mobile robot localization," in Proc. IFAC Int. Symp. Robot Control, SyRoCo, 2000, pp. 97-102.

[12] J. Cortés, S. Martínez, T. Karatas, and F. Bullo, "Coverage control for mobile sensing networks," IEEE Trans. Robot. Autom., vol. 20, no. 2, pp. 243-255, Apr. 2004.

[13] A. K. Das, R. Fierro, V. Kumar, J. P. Ostrowsky, J. Spletzer, and C. Taylor, "A vision-based formation control framework," IEEE Trans. Robot. Autom., vol. 18, no. 5, pp. 813-825, Oct. 2002.

[14] W. E. Dixon, Y. Fang, D. M. Dawson, and T. J. Flynn, "Range identification for perspective vision systems," IEEE Trans. Autom. Control, vol. 48, no. 12, pp. 2232-2238, Dec. 2003.

[15] W. Dong and J. A. Farrell, "Cooperative control of multiple nonholonomic mobile agents," IEEE Trans. Autom. Control, vol. 53, no. 6, pp. 14341448, Jul. 2008.

[16] D. A. Forsyth and J. Ponce, Computer Vision: A Modern Approach. Englewood Cliffs, NJ: Prentice-Hall, 2002.

[17] R. A. Freeman, P. Yang, and K. M. Lynch, "Distributed estimation and control of swarm formation statistics," in Proc. Amer. Control Conf., 2006, pp. 749-755.

[18] R. Hermann and A. Krener, "Nonlinear controllability and observability," IEEE Trans. Autom. Control, vol. AC-22, no. 5, pp. 728-740, Oct. 1977.

[19] J. P. Hespanha, "State estimation and control for systems with perspective outputs," in Proc. 41st IEEE Conf. Decision Control, 2002, vol. 2, pp. 2208-2213.

[20] H. Inaba, A. Yoshida, R. Abdursul, and B. K. Ghosh, "Observability of perspective dynamical systems," in Proc. 39th IEEE Conf. Decision Control, 2000, pp. 5157-5162.

[21] A. Isidori. Nonlinear Control Systems, 3rd ed. New York, NY: SpringerVerlag, 1995.

[22] J. Lin, A. S. Morse, and B. D. O. Anderson, "The multi-agent rendezvous problem. Part 1: The synchronous case," SIAM J. Control Optim., vol. 46, no. 6, pp. 2096-2119, 2007

[23] G. L. Mariottini, F. Morbidi, D. Prattichizzo, G. J. Pappas, and K. Daniilidis, "Leader follower formations: Uncalibrated vision-based localization and control," in Proc. IEEE Int. Conf. Robot. Autom., 2007, pp. 2403 2408.

[24] G. L. Mariottini, G. J. Pappas, D. Prattichizzo, and K. Daniilidis, "Visionbased localization of leader follower formations," in Proc. 44th IEEE Conf. Decision Control, 2005, pp. 635-640.

[25] N. Michael, J. Fink, and V. Kumar, "Experimental testbed for large multirobot teams," IEEE Robot. Autom. Mag., vol. 15, no. 1, pp. 53-61, Mar. 2008.

[26] H. Nijmeijer and A. J. van der Shaft, Nonlinear Dynamical Control Systems. New York: Springer-Verlag, 1990.

[27] R. Olfati-Saber, J. A. Fax, and R. M. Murray, "Consensus and cooperation in networked multi-agent systems," Proc. IEEE, vol. 95, no. 1, pp. 215233, Jan. 2007.

[28] L. E. Parker, "Multiple mobile robot systems," in Handbook of Robotics, B. Siciliano and O. Khatib, Eds. New York: Springer-Verlag, 2008, ch. 40, pp. 921-941.

[29] J. E. Slotine and W. Li, Applied Nonlinear Control. Englewood Cliffs, NJ: Prentice-Hall, 1991.

[30] P. Tabuada, G. J. Pappas, and P. Lima, "Motion feasibility of multiagent formations," IEEE Trans. Robot., vol. 21, no. 3, pp. 387-392, Jun. 2005.

[31] K. H. Tan and M. A. Lewis, "High precision formation control of mobile robots using virtual structures," Auton. Robots, vol. 4, no. 4, pp. 387-403, 1997.

[32] R. Vidal, O. Shakernia, and S. Sastry, "Following the flock: Distributed formation control with omnidirectional vision-based motion segmentation and visual servoing," IEEE Robot. Autom. Mag., vol. 11, no. 4, pp. 14-20, Dec. 2004.

\section{Defining Conditions for Nonsingular Transitions Between Assembly Modes}

\author{
Alfonso Hernandez, Oscar Altuzarra, Victor Petuya, \\ and Erik Macho
}

\begin{abstract}
It is known that there are parallel manipulators that can perform nonsingular transitions between different assembly modes. In particular, 3-degree-of-freedom (DOF) manipulators have received primary attention related to this phenomenon. In this paper, the conditions for the existence of special points in the projection of the direct-kinematic-problemsingularity locus onto the joint space for one constant input are obtained. From these conditions, the coordinates of all cusp points can be obtained analytically. Encircling one of these cusp points, it is possible to make a nonsingular transition between two assembly modes of a parallel manipulator. Utilizing these conditions, the range for the existence of cusp points of each input value can be also determined. An extension of the concept of cusp points to the complete joint space is also performed. The procedure is applied to an $R P R-2-P R R$ parallel manipulator that can be solved analytically. Its dimensional variables are parametrized as a 1-D function, and all results are obtained in closed form, which is a benchmark example for other procedures.
\end{abstract}

Index Terms-Assembly mode, cusp point, nonsingular transition, reduced configuration space.

\section{INTRODUCTION}

The size and shape of the reachable area, which is the workspace, is one of the main design criteria in robots. In the case of parallel manipulators, its geometry is often complex and is divided by internal singularities. Normally, these manipulators have multiple solutions of the direct kinematic problem (DKP) and inverse kinematic problem (IKP). Singularity locus are obtained by the analysis of the conditions that make the Jacobian matrices singular. A common practice in the design of parallel manipulators is to restrict their operational workspace to one of the singularity-free regions.

The workspace boundaries are IKP singularities, where the determinant of the inverse Jacobian $\left|\mathbf{J}_{\mathrm{IKP}}\right|$ is null. At these postures, the control of the robot is not lost, but some manipulability restrictions appear due to the dependency among output velocities. Besides, when the determinant of the direct Jacobian $\left|\mathbf{J}_{\mathrm{D} \mathrm{K}}\right|$ is null, DKP singularities

Manuscript received May 26, 2009; revised July 30, 2009. First published September 29, 2009; current version published December 8, 2009. This paper was recommended for publication by Associate Editor F. Thomas and Editor W. K. Chung upon evaluation of the reviewers' comments. This work was supported by the Spanish Government via the Ministerio de Innovación y Ciencia under Project DPI2008-00159 and the Fondo Europeo de Desarrollo Regional (FEDER) funds of the European Union and the University of the Basque Country under Project GIC07/78-IT-186-07.

The authors are with the Department of Mechanical Engineering, University of the Basque Country, Bilbao 48013, Spain (e-mail: oscar.altuzarra@ehu.es).

This paper has supplementary downloadable multimedia material available at http://ieeexplore.ieee.org, provided by the author. This material includes one video (nonsing_transition.avi) that shows a nonsingular transition between two solutions of the direct position problem of an $R P R-2-P R R$ parallel manipulator. On the right side, the motion over the reduced configuration space surface is shown. Direct kinematic problem singularity curves, where the robot becomes uncontrollable, are avoided, encircling a triple coalescence posture (which appears as a cusp point when it is projected onto the joint space). Simultaneously, on the left side, the corresponding motion of the manipulator is shown. Input variables have same values at the beginning and at the end of the motion. The size of the video is not specified. Contact oscar.altuzarra@ehu.es for further questions about this work.

Color versions of one or more of the figures in this paper are available online at http://ieeexplore.ieee.org.

Digital Object Identifier 10.1109/TRO.2009.2030229 